\title{
ESTUDIO ERGONÓMICO COMO PARTE DE LA RESPONSABILIDAD SOCIAL EN TRABAJADORES DEL CENTRO REGIONAL DE INFORMÁTICA DE LA UNIVERSIDAD VERACRUZANA, MÉXICO ${ }^{1}$
}

\author{
Patricia Martínez Moreno \\ María Guadalupe Aguirre Alemán ${ }^{3}$ \\ Wilfran Donaldo González José e $^{4}$
}

Para citar este artículo: Martínez, P., Aguirre, M. y González, W. (2015). Estudio ergonómico como parte de la responsabilidad social en trabajadores del centro regional de informática de la Universidad Veracruzana, México. Inquietud Empresarial. Vol. XV (2), 87-114.

Fecha de recepción: 07 de septiembre de 2015 Fecha de aceptación: 11 de diciembre de 2015

\footnotetext{
Artículo de investigación científica como resultado del proyecto de investigación titulado "Estudio ergonómico en trabajadores del Centro Regional de Informática como parte de la responsabilidad social" realizado en la Universidad Veracruzana, Campus Coatzacoalcos, México.

2 Maestra en Tecnología Educativa con Doctorado en Educación. Profesora de tiempo completo de la Facultad de Contaduría y administración de la Universidad Veracruzana, Campus Coatzacoalcos, Veracruz, México. patym10@hotmail.com

3 Maestra en Administración con Doctorado en Gobierno y Administración Pública. Profesora de tiempo completo de la Facultad de Contaduría y administración de la Universidad Veracruzana, Campus Coatzacoalcos, México. maguirre_aleman@hotmail.com

4 Estudiante de la Facultad de Contaduría y administración de la Universidad Veracruzana, Campus Coatzacoalcos, Veracruz, México. wilfranglezjose@outlook.com
} 


\title{
Resumen
}

El objetivo de aplicar la Responsabilidad Social Empresarial (RSE) en las Organizaciones es el intento del fomento de la competitividad de las empresas con el aumento de la cohesión social, de la solidaridad y el respeto al trabajador. Cabe señalar que no se tiene una definición única de Responsabilidad Social Empresarial, pero se han desarrollado directrices a nivel internacional como la Organización para la Cooperación y el Desarrollo Económicos (OCDE) y Global Compact, quienes dictaron las primeras líneas directrices para empresas multinacionales. Este trabajo de investigación se centra específicamente en la directriz relación laboral, desde una perspectiva de cuidar y procurar por la salud, a partir del uso de un instrumento aplicado a los trabajadores. Como producto de esta investigación se presentaron recomendaciones y por supuesto se sugirieron algunas acciones preventivas que servirán en el futuro, sin dejar de lado que periódicamente el trabajador debe ser evaluado por mínimo que sea los riesgos en su área de trabajo.

Palabras clave: responsabilidad social empresarial, salud, ergonomía, informática, trabajador.

\section{Ergonomic study as part of social responsibility in workers of the regional center of computing at the Veracruz University, Mexico}

\begin{abstract}
The aim to apply Social and business responsibility (RSE) at the different organizations is the attempt to encourage the competitiveness of the companies with the increasing of the social cohesion, solidarity and respect for the worker. It is important to take into account, that there is not a single definition of Social and business responsibility, but it has developed guidelines at different international levels such as: Organization for Economic Co-operation and Development (OCDE) and the Global Compact, who has established the first guidelines for the multinational companies. This research project is focused on the employment guideline bearing in mind the concern towards the health starting from the use of an instrument applied to the workers. As result of this investigation, it was presented recommendations and it was suggested some preventive actions that will be useful for the future, without forgetting the idea that workers have to be evaluated periodically event the risks at their work areas.
\end{abstract}


EST UDIO ERGONÓMICO COMO PARTE DE LA RESPON SABILIDAD SOCIAL EN TRABAJADORES DEL CENTRO REGIONAL DE INFORMÁTICA DE LA UNIVERSIDAD VERACRUZANA, MÉXICO

Key words: corporate social responsibility, competitiveness, health, ergonomics, computing.

\section{Estudo ergonômico como parte da responsabilidade social na regional centro de trabalhadores computação Universidade Veracruzana, México}

\section{Resumo}

O objetivo de aplicar a Responsabilidade Social Empresarial (RSE) nas Organizações é o intuito de fomento da competitividade das empresas com o aumento da coesão social, da solidariedade e o respeito ao trabalhador. Cabe sinalizar que não se tem uma definição única de Responsabilidade Social Empresarial, mas se tem desenvolvido a nível internacional como a Organização para a Cooperação e o Desenvolvimento Econômico (OCDE) e Global Compact, quem ditaram as primeiras linhas de diretrizes para as empresas multinacionais. Este trabalho de investigação centra-se específicamente na diretriz relação laboral, desde uma perspectiva de cuidar e procurar por saúde, a partir do uso de um instrumento aplicado aos trabalhadores. Como produto desta investigação apresentaram-se recomendações e sem dúvidas surgiram algumas ações preventivas que sirvam no futuro, sem deixar do lado que periódicamente o trabalhador deve ser avaliado por no mínimo que seja os riscos na sua área de trabalho.

Palavras chave: responsabilidade social empresarial, competitividade, saúde, ergonomia, informática. 


\section{Introducción}

La Responsabilidad Social Empresarial (RSE) en las Organizaciones busca reconciliar el fomento de la competitividad de las empresas con el aumento de la cohesión social, de la solidaridad y el respeto hacia el trabajador. Se carece de una definición única de Responsabilidad Social Empresarial (RSE) o Responsabilidad Social Corporativa (RSC), pero se han desarrollado directrices a nivel internacional como la Organización para la Cooperación y el Desarrollo Económicos (OCDE) y Global Compact, quienes dictaron las primeras líneas directrices para empresas multinacionales; y la Organización de las Naciones Unidas (ONU) que desarrolló directrices cuya finalidad es impulsar la RSE, además de pretender evitar las consecuencias negativas de la globalización. Estás directrices actúan como principios básicos y generales que guían el correcto desempeño de las compañías en su vinculación con la sociedad y son cinco: ética y gobierno corporativo, relación laboral, proveedores y clientes, medio ambiente, gobierno y sociedad.

Este trabajo de investigación muestra los resultados de indagar sobre la ergonomía en los trabajadores del Centro Regional de Informática de la Unidad de Servicios Bibliotecarios y de Información (USBI) en la Universidad Veracruzana, Campus Coatzacoalcos México, a partir de un cuestionario validado. El objetivo de esta investigación es identificar si en los trabajadores se aplican medidas ergonómicas de iluminación y mobiliario en el uso del equipo de cómputo.

Al partir de la definición de la ISO 26000, la Responsabilidad Social (RS) es la "responsabilidad de una organización ante los impactos que sus decisiones y actividades ocasionan en la sociedad y en el medio ambiente, mediante un comportamiento ético y transparente que contribuya al desarrollo sostenible, incluyendo la salud y el bienestar de la sociedad; tome en consideración las expectativas de las partes interesadas; cumpla con la legislación aplicable y sea coherente con la normativa internacional de comportamiento, y esté integrada en toda la organización y se lleve a la práctica en sus relaciones”. (Argandoña, 2012).

Según Caravedo (1999), entendemos por Responsabilidad Social Empresarial (RSE) "una filosofía corporativa adoptada por la alta dirección de la empresa para actuar en beneficio de sus propios trabajadores, sus familias y el entorno social en las zonas de influencia de las empresas".

La RSE es aquella que debe procurar por la salud, bienestar de la sociedad y que sea coherente a la normativa. La literatura indica que existen diversos estudios de RSE 
EST UDIO ERGONÓMICO COMO PARTE DE LA RESPONSABILIDAD SOCIAL EN TRABAJADORES DEL CENTRO REGIONAL DE INFORMÁTICA DE LA UNIVERSIDAD VERACRUZANA, MÉXICO

enfocados a la ética y gobierno corporativo, medio ambiente, proveedores y clientes; pero también existen estudios enfocados a los trabajadores.

Para Correa, Gallopin y Núñez (2005), las empresas están conscientes del creciente desafío de cuantificar el impacto que sus actividades, procesos, productos y servicios tienen sobre el medio ambiente y el entorno social.

Con base en lo anterior, este estudio se enfoca principalmente en el trabajador, desde el cuidado de su salud desde la ergonomía informática, específicamente se analiza el sistema visual y sistema muscular desde el trabajo de una oficina frente a una computadora. Para ello, fue necesario en principio hacer una revisión bibliográfica que sustente dicho propósito, identificar variables y recopilar información del área de estudio. Es un tema que en ocasiones puede pasarse por alto dentro de la Responsabilidad Social, por lo que se decidió analizarlo desde la perspectiva relación laboral. En ocasiones los trabajadores en este caso, los empleados del CRI también carezcan de la información teórica como práctica de las repercusiones en cuanto a su salud por una mala ergonomía informática; de tal manera la pregunta es: ¿Qué impacto puede tener la ergonomía informática en la salud de los trabajadores de una organización?

\section{Referentes teóricos}

\subsection{Ergonomía}

La palabra ergonomía se deriva de las palabras griegas "ergon", que significa trabajo, y "nomos", que significa leyes; por lo tanto, ergonomía significa "leyes del trabajo" (Rodríguez, 2013).

Según el Consejo Gallego de Cooperativas, a lo largo del desarrollo de la civilización humana, y especialmente en el ámbito industrial, el desarrollo y selección de herramientas y maquinaria ha llevado un proceso continuo, buscando mejorar la eficiencia y efectividad de la producción, sin embargo, este desarrollo no siempre ha resultado en mejores condiciones de trabajo, seguridad y comodidad para quienes los utilizan.

El trabajo perjudicial es el que realizamos cuando no hacemos un uso correcto o apropiado de las fuerzas y facultades que nos han sido concedidas, y que lleva al deterioro de las cosas y las personas. El trabajo útil (eficaz, diríamos hoy en día) es aquel que consigue que el desarrollo de nuestra actividad profesional no sea algo 
meramente mecánico o externo, sino que se vaya perfeccionando con la práctica, superando una serie de niveles internos sensorial, intelectual y espiritual, hasta alcanzar la felicidad por medio del trabajo. (Leirós, 2009).

En el siglo XIX con el descubrimiento de la máquina de vapor, la interacción hombremáquina estaba supeditada absolutamente a la experiencia; hoy en día no se puede basar dicha interacción solamente en el sentido común, la intuición o la experiencia. El término ergonomía fue propuesto por el naturalista Polaco Woitej Yastembowsky en 1857 en su estudio Ensayos de Ergonomía o Ciencias del Trabajo, basado en las leyes objetivas de la ciencia sobre la naturaleza, propuesto por Mario Jaureguiberry, en la cual se proponían construir un modelo de la actividad laboral humana.

Por otra parte, fue hasta la segunda guerra mundial que la ergonomía mostró un desarrollo importante, como la atención estaba centrada en el desarrollo de máquinas tales como aviones, equipo de comunicaciones entre otros equipos, operadores y encargados de mantenimiento presentaron problemas, pues poseían conocimientos escasos sobre estas nuevas máquinas. Muchos de los problemas que se presentaron fueron consecuencia del desconocimiento del comportamiento humano y las características físicas del usuario (Cuenca, 2012).

En Oxford, Inglaterra el 12 de julio de 1949 K.F.H. Murrel, creo el término "ergonomía", acuñado de las raíces griegas "ergon": trabajo y nomos: leyes, y fue adoptado oficialmente durante la creación, en julio de 1949, de la primera sociedad de ergonomía, la Ergonomics Research Society, fundada por ingenieros, fisiólogos y psicólogos británicos con el fin de adaptar al trabajo al hombre. (Rodríguez, 2013)

El término ergonomía empezó a utilizarse alrededor de 1950, cuando las prioridades de la industria en desarrollo comenzaron a anteponerse a las prioridades de la industria militar. Singleton (1982) describe detalladamente el desarrollo de la investigación y sus aplicaciones, a lo largo de los 30 años siguientes. Algunas organizaciones de las Naciones Unidas, en especial la OIT y la OMS, comenzaron su actividad en este campo en el decenio de 1960.

En agosto del año 2000, la Asociación Internacional de Ergonomía (IEA), definió a la ergonomía, o factores humanos, como la disciplina científica concerniente con el estudio de las interacciones entre los humanos y otros elementos de un sistema, así como la profesión que aplica la teoría, principios, datos y métodos al diseño, en orden de optimizar el bienestar humano y el desempeño general del sistema. 
EST UDIO ERGONÓMICO COMO PARTE DE LA RESPONSABILIDAD SOCIAL EN TRABAJADORES DEL CENTRO REGIONAL DE INFORMÁTICA DE LA UNIVERSIDAD VERACRUZANA, MÉXICO

Todo lo anterior se dio como consecuencia del esfuerzo excesivo y del estrés de la batalla, de la complejidad técnica de los nuevos equipos de guerra, por lo que era necesario adaptar el trabajo al hombre, esto es, diseñar un equipo en función de la capacidad y limitaciones del individuo. Entre 1963 y 1964 se formula en Inglaterra, la tesis del enfoque sistémico en la ergonomía, cuyo máximo representante fue W. Singletón. (Melo, 2003).

1.1.1. Concepto. El término ergonomía se deriva de las palabras griegas "ergon" que significa trabajo, y "nomos", leyes naturales, por lo que literalmente significa "leyes del trabajo", y podemos decir que es la actividad de carácter multidisciplinar que se encarga del estudio de la conducta y las actividades delas personas, con la finalidad de adecuar los productos, sistemas, puestos de trabajo y entornos a las características, limitaciones y necesidades de sus usuarios, buscando optimizar su eficacia, seguridad y confort (Laurig et al, 1992).

La ergonomía influye sobre el ser humano, por lo que debe considerar el aspecto físico, cognitivo, social, organizacional, ambiental, y cualquier otro factor que tenga influencia y que resulte relevante. Este amplio panorama que cubre la ergonomía puede clasificarse en forma general en tres grandes áreas, que son:

1.1.2. Ergonomía física. En la ergonomía física están involucrados principalmente los especialistas en las áreas de anatomía, antropometría, características fisiológicas y biomecánicas aplicadas a la actividad física del humano, así como el análisis de los factores ambientales y su influencia sobre el desempeño de los humanos.

La ergonomía física es la referida a las características anatómicas, Antropométricas, fisiológicas y biomecánicas en relación con la actividad física. (Delgado, 2011).

1.1.3. Ergonomía cognitiva. La ergonomía cognitiva estudia las interacciones entre un dispositivo, objeto, producto y un individuo. Estas interacciones hacen uso de las principales funciones mentales (percepción, memoria, razonamiento). La ergonomía cognitiva se ocupa entonces de los problemas de costo cognitivo derivado de esta interacción. (Cañas y Salmerón, 2004).

1.1.4. Ergonomía organizacional. La ergonomía organizacional se interesa por la optimización de los sistemas socio-técnicos, esto incluye su estructura organizacional, sus reglas y sus procesos. Los temas pertinentes comprenden la comunicación, la gestión de los recursos del colectivo, la concepción del trabajo, la concepción de los 
horarios de trabajo, el trabajo en equipo, la concepción participativa, la ergonomía comunitaria, el trabajo cooperativo, las nuevas formas de trabajo, la cultura organizacional, las organizaciones virtuales, el teletrabajo y la gestión por la calidad (Carrasquero y Seijo, 2009).

La Ergonomía es una disciplina autónoma basada en resultados de estudios empíricos y que pueden proporcionar informaciones ciertas para modificar instalaciones, maquinarias, equipos, herramientas y dispositivos en general, así como la tecnología y los procesos para adaptar mejor el trabajo al hombre (Jaureguiberry, s/f).

De acuerdo con la Medicina Laboral y la Organización Internacional del Trabajo, O.I.T., la Medicina del Trabajo es el "Fomentar y mantener el más elevado nivel de bienestar físico, mental y social de los trabajadores en todas sus profesiones. Prevenir todo daño causado a la salud de estos por las condiciones del trabajo. Protegerlos en sus empleos contra los riesgos resultantes de la presencia de agentes nocivos para la salud".

Así bien, se observa que los principios de la Medicina y de la Ergonomía son afines, aunque sus caminos sean otros. La definición de Trabajo en el sentido de la Ergonomía es: la totalidad de energía o información que es transformada o elaborada por el hombre durante el cumplimiento de las tareas laborales.

Objetivos de la ergonomía según el Instituto Nacional de Seguridad e Higiene en el Trabajo (INSHT, s.f):

- Seleccionar la tecnología más adecuada al personal disponible.

- Controlar el entorno del puesto del trabajo.

- Detectar los riesgos de fatiga física y mental.

- Analizar los puestos de trabajo para definir los objetivos de la formación.

- Optimizar la interrelación de las personas disponibles y la tecnología utilizada.

- Favorecer el interés de los trabajadores por la tarea y por el ambiente de trabajo.

1.1.5. Ergonomía informática. La ergonomía informática es una ciencia moderna que se encarga de estudiar todo lo relacionado al bienestar, comodidad y seguridad de los usuarios de sistemas computacionales. Además, permite analizar la influencia y repercusiones de dichos sistemas y del ambiente laboral en la salud de los usuarios, así como en su productividad. (Ramos, 2007). 
EST UDIO ERGONÓMICO COMO PARTE DE LA RESPONSABILIDAD SOCIAL EN TRABAJADORES DEL CENTRO REGIONAL DE INFORMÁTICA DE LA UNIVERSIDAD VERACRUZANA, MÉXICO

\subsection{La ergonomía en los espacios de trabajo}

El puesto de trabajo, es el área en el que el empleado desempeña sus tareas diarias. Puede o no ser ocupado todo el tiempo o simplemente ser uno de los lugares en donde se realiza el trabajo. Es de vital importancia que el puesto de trabajo cuente con un buen diseño para evitar problemas de salud relacionados con condiciones laborales ineficientes. Todo puesto de trabajo debe ser diseñado teniendo en cuenta al trabajador y las tareas que este desempeña con el fin de que se realicen cómodamente y sin ningún problema.

Si el puesto de trabajo está diseñado correctamente, el empleado mantendrá una posición corporal adecuada, lo que es importante ya que de no ser así puede ocasionar múltiples problemas.

Mobiliario. El adecuado mobiliario de oficina tal como silla, escritorio, monitor y entre otros componentes son de vital importancia en el área de trabajo, puesto la correcta postura y el uso de estos influyen sobre la salud de los usuarios, por lo tanto, deben de tomarse algunas precauciones y recomendaciones en el uso de las misma.

Silla. Existen una variedad inmensa de sillas, pero todas y cada una de estas están diseñadas para diferentes tipos de trabajo y de uso, por esto es fundamental que sea de altura regulable para respetar los ángulos de 90 grados en cadera, rodilla y tobillo, debe tener apoyo en raquis lumbo-dorsal para relajar la espalda y disminuir la presión entre los discos intervertebrales y respetar un espacio detrás de las rodillas para no irrumpir la circulación en los miembros inferiores. Y preferentemente debe ser giratoria, con apoya brazos y con rueditas, para facilitarnos el movimiento dentro de la oficina.

Monitor. Deberá estar puesto justamente frente al usuario donde la parte superior del mismo, quede situado a la altura de los ojos, para tener una correcta postura de la cabeza y evitar inclinaciones. La postura correcta esta entre los $35^{\circ}$ y $40^{\circ}$, mientras que el del monitor deberá tener una postura de entre $10^{\circ}$ a $20^{\circ}$. (Fidalgo, 2006).

Escritorio. Debe tener un espacio para las piernas de aproximadamente $60 \mathrm{~cm}$ de longitud, o un espacio entre la tabla y las rodillas. Tendrá una altura media de $70 \mathrm{~cm}$ y nos permitirá tener el mouse paralelo al teclado. Los brazos de la silla no deberán tropezar con el escritorio, esto para evitar una posición forzada y deberá estar a la altura del codo (Ergocare, 2011). 
Teclado y Mouse. Brakamonte citado en ARMS (2012) plantea que, éstos deben estar altura de los codos, de esta manera se evita posiciones forzadas, de la misma manera el teclado deberá estar a la altura de las muñecas, hacer uso del teclado en una posición inadecuada puede provocar problemas en la columna del usuario. De la misma manera, el mouse debe situarse de tal forma en el que se pueda hacer uso de él con libertad y facilidad.

Iluminación. La iluminación es la cantidad de luz que el usuario necesita, no se trata de la luminosidad en general, sino solo la necesaria en el punto focal del trabajo. Prevención de reflujo, resplandor o reflexión, trata de la descripción de las fuentes ajenas a la luz dentro del punto focal del trabajo visual, que es en gran medida mayor a la luminosidad del monitor, que produce disminución en la legibilidad y puede derivar en problemas de salud como fatiga visual y cansancio en los ojos.

Para evitar el reflujo debe tomarse en cuenta lo siguiente:

- Ubicar correctamente los equipos para evitar el reflejo directo.

- Procure obtener un nivel de luminosidad similar a la del monitor.

- Elegir una mesa de manera que se reduzcan los reflejos.

Ventilación. La ventilación es factor que influye directamente en el bienestar y la ejecución de las tareas. Estas pueden verse afectadas por el calor adicional debido a los equipos que a continuación se enuncian, los principales parámetros térmicos y la forma de adaptarlos para conseguir un ambiente térmico adecuado, que no tenga efectos adversos para el confort y la salud.

Temperaturas y velocidad del aire. La temperatura es una variable de gran importancia en el confort y satisfacción de los trabajadores. La percepción de frío o de calor excesivos en oficinas y despachos genera malestar y puede producir estrés. De ahí que se recomienden intervalos de confort, para asegurar el bienestar. Por otro lado, el confort térmico depende de la asimetría de la temperatura radiante, es decir, de la diferencia de la temperatura radiante de las superficies del entorno. (Ramos, 2007).

Por otra parte, la velocidad del aire es un factor importante ya que puede afectar la sensación térmica, a su vez, la sensación térmica depende de la temperatura y velocidad del aire, es por ello que los sistemas de ventilación deberían ser configurados considerando a las personas que laboran en el lugar. La Universidad Complutense de Madrid recomienda que la temperatura sea mantenida dentro de los siguientes rangos: 
EST UDIO ERGONÓMICO COMO PARTE DE LA RESPONSABILIDAD SOCIAL EN TRABAJADORES DEL CENTRO REGIONAL DE INFORMÁTICA DE LA UNIVERSIDAD VERACRUZANA, MÉXICO

- En época de verano... $23^{\circ} \mathrm{C}$ a $26^{\circ} \mathrm{C}$.

- En época de invierno...20 $20^{\circ} \mathrm{C}$ a $24^{\circ} \mathrm{C}$.

Por último, según la Universidad Complutense de Madrid el síndrome del edificio enfermo se presenta en trabajadores que laboran en edificios que casi siempre tiene un sistema de ventilación forzada, generalmente común a todo el edifico o a amplios sectores y donde existe recirculación parcial del aire. Algunos de los síntomas presentados son: sensación de sequedad en membranas mucosas y piel, ronqueras, erupciones cutáneas, dolor de cabeza e infecciones respiratorias.

\subsection{Problemas de salud en trabajadores generados por riesgos ergonómicos en el area laboral}

- Sistema visual. Del $80 \%$ de información que el cuerpo humano recibe, es por medio de la visión, por ello es de suma importancia tomar en cuenta las medidas necesarias para evitar problemas visuales futuros. Esto no causa un daño permanente den los ojos, pero, deben atenderse o tomar las medidas necesarias para que no empeoren con el paso del tiempo. Problemas de salud provocados por el uso prolongado del monitor: daño a la visión, resequedad ocular, fatiga ocular, visión borrosa. (Salud 180, 2013)

Recomendaciones Según la Academia Americana de Oftalmología: Sentarse a una distancia aproximada de 25 pulgadas de la pantalla de la computadora y coloque la pantalla de tal forma que pueda mirarla levemente hacia abajo, reducir el brillo de la pantalla iluminando el área de trabajo adecuadamente, y use un filtro para pantallas si es necesario, usar la regla "20-20-20": cada 20 minutos, eche un vistazo a un objeto que esté a unos 20 pies de distancia, por unos 20 segundos y hacer uso de lágrimas artificiales para refrescar los ojos cuando los sienta secos.

- Sistema muscular. Al sentarse se debe no solo adoptar la postura más cómoda, sino que además tener presentes un grupo de factores tales como: acomodo de la espalda, manos y pies.

La elección de un mobiliario adecuado, la colocación correcta de los elementos y una buena postura de trabajo pueden reducir al mínimo las molestias derivadas de este trabajo. (Rodríguez, 2013). 


\subsection{Problemas musculares derivados del uso de equipos de cómputo}

- Dolor de cuello y espalda: es originada por pasar un tiempo prolongado en sillas poco cómodas. Pero no sólo esto la origina, también la posición del monitor, ya que, si esta no está en la posición adecuada en cuanto a nuestro campo visual, eso provoca que forcemos los músculos del cuello y la espalda. El mobiliario de oficina debe tener respaldada la columna vertebral desde el inicio hasta su base. Los problemas de salud musculares pueden ser también derivados de las malas posturas que el usuario puede tomar en las sillas.

- Dolor de muñeca: es causado por realizar trabajo repetitivo, y una de estas es trabajar con la computadora en el caso de escribir en el teclado y utilizar el ratón. Estas acciones repetitivas, al parecer inofensivas suelen afectar después de muchas sesiones de largas horas.

Una de las lesiones más frecuentes por movimientos repetitivos es síndrome el túnel carpiano. Éste se produce cuando el nervio que va del antebrazo a la mano (y, para ello, atraviesa el 'túnel' de la muñeca), se comprime como consecuencia de la hinchazón y la inflamación de los tendones y ligamentos (Del Rio y González, 2007).

\section{Reporte de investigación}

\subsection{Planteamiento del problema}

Las organizaciones públicas y privadas realizan grandes esfuerzos por mejorar la operatividad de sus sistemas de información, incorporando equipos de cómputo modernos o actualizando los que ya se tienen en operación, sin embargo, hay una parte importante que muchas de estas empresas han pasado por alto, la importancia de trabajar con computadoras en un ambiente ergonómico adecuado. La selección del equipo informático en un ambiente laboral adecuado ayuda a prevenir alteraciones visuales, musculares y esqueléticas, con ello se busca que el trabajador reciba la formación e información suficiente de cómo puede utilizar y ajustar los equipos con los que trabaja. Desde la década de 1990, han aparecido publicaciones relacionadas con el uso de computadores y problemas musculo-esqueléticos, tales como tensiones musculares o sobrecargas músculos tendinosos en miembros superiores y en las zonas cervicales, dorsales y lumbares de la columna.

La aplicación de la tecnología de las computadoras, acompañadas del uso de monitores revolucionó los espacios laborales en muchos lugares del mundo. Por ello, resulta frecuente encontrar problemas de salud, particularmente aquéllos relacionados con 
EST UDIO ERGONÓMICO COMO PARTE DE LA RESPON SABILIDAD SOCIAL EN TRABAJADORES DEL CENTRO REGIONAL DE INFORMÁTICA DE LA UNIVERSIDAD VERACRUZANA, MÉXICO

la vista y el sistema músculo-esquelético (SME, MSS: musculoskeletal system), dentro de la población trabajador. Por ejemplo, en el estudio de Bongers se indica que quienes trabajan directamente con monitores o unidades de video (VDU: visual display unit o VDT: visual display terminal) muestran prevalencias altas $(61.5 \%)$ de dolor en cuello y hombros que pueden interferir en sus actividades laborales; debido al uso cada vez más extenso de las computadoras se han realizado estudios que intentan responder las preguntas acerca de la seguridad y la salud de los usuarios de monitores de computadora.

Algunos estudios muestran que el problema más frecuente entre los usuarios de monitores de computadora corresponde a la visión, estos padecimientos visuales parecen incrementarse con rapidez, ya que se ha estimado que el $90 \%$ de los trabajadores que utilizan la computadora por más de 3 horas al día los experimentan de alguna forma; si bien son relativamente pocas las publicaciones que reportan la incidencia de trastornos musculo esqueléticos entre los usuarios de computadoras. En Finlandia, por ejemplo, se reportó una incidencia anual del 34\%, mientras que en Estados Unidos fue de 58\%, lo cual representa para este país un 30\% del total de los casos de patología laboral. Por su parte, Ariëns et al señalan que permanecer sentado por más del $95 \%$ de la jornada laboral es un factor de riesgo para el dolor en el cuello (Del Rio y González, 2007).

En la mayor parte de los casos, la postura que adoptan los usuarios de equipo de cómputo conduce a la aparición de males prematuros que representan, en general, el $75 \%$ de las lesiones ergonómicas y son la primera causa de incapacidad registrada en el Instituto Mexicano de Seguro Social: cefaleas, dolores de espalda, molestias cervicales, lumbalgias, agarrotamientos musculares... los esfuerzos de mano, muñeca, codo y hombro traen consigo tendinitis, tenosinovitis, etc., a consecuencia de movimientos rápidos, forzados y repetidos que inflaman las articulaciones. Denominado "Síndrome de Tensión Repetitiva" y herencia de los adelantos tecnológicos, conforma un cuadro clínico estrechamente ligado al manejo de video terminales.

Teniendo conocimiento de las consecuencias que un ambiente de trabajo anti ergonómico de estos estudios, el problema central de la investigación se define del siguiente modo: ¿Trabajadores de instituciones como el Centro Regional de Informática de la Universidad Veracruzana Campus Coatzacoalcos aplican medidas ergonómicas en su entorno laboral?, ¿Cuentan con recursos de trabajo diseñados ergonómicamente?, ¿Los recursos laborales están diseñados bajo los criterios ergonómicos? 


\subsection{Objetivo general}

Identificar si en los trabajadores del Centro Regional de Informática se aplican medidas ergonómicas de iluminación y mobiliario en el uso del equipo de cómputo, como parte de la Responsabilidad Social Empresarial y las condiciones laborales.

\subsubsection{Objetivos específicos}

- Analizar las diferentes formas de trabajo con relación a la postura y los tiempos de permanencia del grupo estudiado.

- Determinar las condiciones ergonómicas en cuanto a la iluminación y mobiliario en los trabajadores del Centro Regional de Informática.

- Identificar oportunidades de mejora factibles de implementarse, encaminadas a minimizar los factores de riesgo ergonómico en puestos de trabajo con equipo de cómputo.

- Proporcionar alternativas que puedan apoyar y guiar al correcto desempeño del CRI en Responsabilidad Social Empresarial en la directriz relación laboral como medida ergonómica de sus trabajadores.

\subsection{Justificación}

Según Muñoz y Vanegas (2012) "Los reportes en la literatura científica sugieren que los usuarios de computadores poseen tasas elevadas de desórdenes musculo esqueléticos y de visión al compararlos con los no usuarios, particularmente entre un $11 \%$ y $14 \%$ de trabajadores ven limitadas sus actividades debido al dolor de cuello, mostrando la evidencia que intervenciones en las que se modificó los puestos de trabajo y la postura del trabajador aún no muestran efectividad en la reducción de la prevalencia de este problema".

Una vez expuesta esta información surgió la necesidad de realizar esta investigación en dicho centro, con la finalidad de contribuir con la RSE a que las organizaciones al momento de realizar planes de construcción y diseño de oficinas, áreas con equipos de cómputo, considere dos aspectos importantes: la ergonomía y el cuidado de la salud del trabajador.

Con ello pueden resultar beneficiados el trabajador, la productividad de la empresa, esto debido a que, en cuestión de energía eléctrica, la organización podrá economizar 
EST UDIO ERGONÓMICO COMO PARTE DE LA RESPONSABILIDAD SOCIAL EN TRABAJADORES DEL CENTRO REGIONAL DE INFORMÁTICA DE LA UNIVERSIDAD VERACRUZANA, MÉXICO

en consumos de energía, y al cuidar la salud del usuario, permitirá que estos, laboren de manera eficaz y las probabilidades que estos se ausenten de sus jornadas laborales sean menores, lo que significa que no se exponen cursos o planes de trabajo ya previamente establecidos por la organización.

\section{Metodología}

El trabajo de investigación tiene un enfoque cuantitativo, el cual aplica un proceso secuencial y probatorio además de plantear una situación delimitada y las preguntas de investigación son específicas. La recolección de datos se fundamenta en la medición y los fenómenos estudiados deben poder observarse o referirse en el "mundo real", y se generalizan los resultados encontrados en un segmento o la muestra. El alcance de la investigación es completamente descriptivo porque se muestran las propiedades y características ergonómicas de los trabajadores en las áreas laborales del CRI con la finalidad de emitir recomendaciones y evitar ciertos padecimientos derivados de ello. Transaccional por que la recolección de la información se lleva a cabo en un solo momento.

Con base en la literatura se diseña un cuestionario con 10 ítems, los cuales tienen como finalidad el poder recabar información para su futuro análisis, de esta forma se podrá indagar y conocer si se aplican medidas ergonómicas en los trabajadores del CRI en cuanto a iluminación y mobiliario con la finalidad de proporcionar alternativas que puedan apoyar y guiar al correcto desempeño en Responsabilidad Social Empresarial en la directriz relación laboral. No hay reglas universales, ni siquiera consenso entre los investigadores. En estudios que contienen análisis de datos cuantitativos, la opción más común es incluir la o las hipótesis de investigación únicamente (Degelman et al, 2005).

\subsection{Hipótesis}

H1: En los trabajadores del Centro Regional de Informática se aplican medidas ergonómicas de iluminación y mobiliario en el uso del equipo de cómputo como parte de la Responsabilidad Social en el cuidado de la salud laboral.

\subsection{Pregunta de investigación}

¿Se aplican medidas ergonómicas de iluminación y mobiliario en los trabajadores del CRI cuando hacen uso del equipo de cómputo como parte de la Responsabilidad Social en el cuidado de la salud laboral? 


\subsection{Universo de estudio}

El estudio que se presenta, se enfoca en el personal administrativo del Centro Regional de Informática, el cual brindó las facilidades para considerarlo como área de estudio. Dicho Centro es una Unidad Académica de la Universidad Veracruzana, que realiza actividades de préstamos de equipo para clases y prácticas individuales, responsables de la red institucional, dan el servicio de apoyo a los problemas técnicos de las facultades del mismo campus en cuanto a: redes, telefonía IP, enlaces de videoconferencias, sistemas universitarios, entre otras.

Para tal estudio se necesita la participación de la totalidad de los trabajadores de dicho centro el cual sólo son 8 . El estudio se lleva a cabo mediante un cuestionario el cual está conformado por 10 ítems, los cuales tienen como finalidad de recabar información para identificar si en el centro regional de informática se aplican medidas ergonómicas en el mobiliario e iluminación en el uso del equipo de cómputo.

\subsection{Variables de estudio y técnicas e instrumentos}

- Trabajador

- Mobiliario

- Iluminación

Se empleó un cuestionario diseñado por los autores y expertos en el área, para identificar las medidas ergonómicas relacionadas con el uso de equipos de cómputo en el entorno laboral desde dos dimensiones: iluminación y mobiliario. Se tomó como método de medición el cuestionario puesto que el mismo está conformado por preguntas que pueden dar respuesta a una o más variables a medir. El cuestionario está estructurado en dos segmentos, aquellos ítems que miden el sistema visual en la dimensión iluminación y los que miden el sistema muscular en la dimensión mobiliario manera que, cada pregunta al ser contestada, logre facilitar los conocimientos requeridos acerca de las condiciones ergonómicas en el Centro Regional de Informática de la Universidad Veracruzana.

En primera instancia, se realizó una prueba piloto, la cual los participantes respondieron el cuestionario on-line, dado que no se obtuvo la cantidad de respuestas esperadas, se optó por aplicar el cuestionario de manera física, impreso en hojas recicladas a todos los trabajadores del CRI. Con los resultados obtenidos de una prueba piloto, se logró calcular la confiabilidad con el que el instrumento cuenta, es decir el alfa de Cronbach ya que mediante el análisis de fiabilidad se determinó si nuestro instrumento de medición es correcto y que tan conciso es. Para lo anterior, se 
llevó a cabo por medio del programa IBM Statics y los resultados fueron lo que se muestra en el cuadro siguiente:

Tabla 1. Coeficiente Alfa de Cronbach

\begin{tabular}{|c|c|}
\hline \multicolumn{2}{|c|}{ ESTADÍSTICOS DE FIABILIDAD } \\
\hline Alfa de Cronbach & Elementos \\
\hline 0.85 & Todos los trabajadores \\
& 8 \\
\hline
\end{tabular}

Fuente: elaboración propia

De esta forma constatamos que nuestro instrumento estaba adecuado de forma correcta y a se podía proceder a su aplicación. El criterio de confiabilidad del instrumento, se determinó, por el Coeficiente Alfa de Cronbach, requiere de una sola administración del instrumento de medición y produce valores que oscilan entre cero y uno. En el siguiente cuadro muestra el total y número de preguntas que abarca cada categoría de estudio.

Tabla 2. Dimensiones

\begin{tabular}{|c|c|}
\hline Dimensiones & Ítems \\
\hline Iluminación & $3,4,5,10$ \\
\hline Mobiliario & $6,7,8,9$ \\
\hline
\end{tabular}

Fuente: elaboración propia

La orientación de los ítems para dicho cuestionario fue de manera positiva, y con base a la escala de Likert. Es aplicable a escalas de varios valores posibles, por lo que puede ser utilizado para determinar la confiabilidad en escalas cuyos ítems tienen como respuesta más de dos alternativas. Se tomó como referencia la siguiente escala:

Tabla 3. Escala de Likert utilizada en el cuestionario.

\begin{tabular}{|l|}
\hline \multicolumn{1}{|c|}{ Valor } \\
\hline 5.- Definitivamente Sí \\
\hline 4.- Probablemente Sí \\
\hline 3.- Indeciso \\
\hline 2.- Probablemente No \\
\hline 1.-Definitivamente No \\
\hline
\end{tabular}

Fuente: Likert 
Antes de recolectar los datos reales para esta investigación, se realizaron las gestiones necesarias con las autoridades de la unidad académica del Centro Regional de Informática.

\section{Resultados y análisis de los datos}

A continuación, se presentan los resultados obtenidos con base en el instrumento respondido por los trabajadores del Centro Regional de Informática representados en tablas.

Tabla 4. Género

\begin{tabular}{|ll|c|c|c|c|}
\hline & Frecuencia & Porcentaje & $\begin{array}{c}\text { Porcentaje } \\
\text { válido }\end{array}$ & $\begin{array}{c}\text { Porcentaje } \\
\text { acumulado }\end{array}$ \\
\hline Válidos & Masculino & 7 & 70.0 & 87.5 & 87.5 \\
& Femenino & 1 & 10.0 & 12.5 & 100.0 \\
Perdidos & Total & 8 & 80.0 & 100.0 & \\
Total & Sistema & 2 & 20.0 & & \\
\hline
\end{tabular}

Fuente: elaboración propia

En la tabla anterior se aprecian las frecuencias en donde 7 de los encuestados son hombres que representa el $87.5 \%$ por lo tanto sólo una mujer labora en dicha institución esta representa solo el $12.5 \%$ de los encuestados.

Tabla 5. Grado de conocimiento ¿conoce el significado del término ergonomía?

\begin{tabular}{|ll|c|c|c|c|}
\hline & Frecuencia & Porcentaje & $\begin{array}{c}\text { Porcentaje } \\
\text { válido }\end{array}$ & $\begin{array}{c}\text { Porcentaje } \\
\text { acumulado }\end{array}$ \\
\hline Válidos & Definitavamente sí & 5 & 50.0 & 62.5 & 62.5 \\
& Probablemente sí & 2 & 20.0 & 25.0 & 87.5 \\
Definitavamente no & 1 & 10.0 & 12.5 & 100.0 \\
Perdidos & Total & 8 & 80.0 & 100.0 & \\
Total & Sistema & 2 & 20.0 & & \\
& & 10 & 100.0 & & \\
\hline
\end{tabular}

Fuente: elaboración propia

En la tabla 5 se puede apreciar que la mayoría de los encuestados sí conoce el significado del término Ergonomía, según la columna de frecuencias. Ya que se les es familiar el tema, pues en teoría, trabajan en un área en donde este tipo de información circula constantemente. La mayoría es representada por el $62.5 \%$, ya que respondieron "Definitivamente que sî": Por otra parte, sólo una persona dijo no conocer el término, éste representa tan sólo el $12.5 \%$ de los encuestados 
EST UDIO ERGONÓMICO COMO PARTE DE LA RESPONSABILIDAD SOCIAL EN TRABAJADORES DEL CENTRO REGIONAL DE INFORMÁTICA DE LA UNIVERSIDAD VERACRUZANA, MÉXICO

Tabla 6. Jornada laboral: cuándo llega su descanso, o se toma un receso ¿siente molestia como irritación, tensión, visión borrosa o enrojecimiento ocular?

\begin{tabular}{|ll|c|c|c|c|}
\hline & Frecuencia & Porcentaje & $\begin{array}{c}\text { Porcentaje } \\
\text { válido }\end{array}$ & $\begin{array}{c}\text { Porcentaje } \\
\text { acumulado }\end{array}$ \\
\hline Válidos & Definitavamente sí & 1 & 10.0 & 12.5 & 12.5 \\
& Probablemente sí & 3 & 30.0 & 37.5 & 50.0 \\
& Probablemente no & 3 & 30.0 & 37.5 & 87.5 \\
& Definitavamente no & 1 & 10.0 & 12.5 & 100.0 \\
Perdidos & Total & 8 & 80.0 & 100.0 & \\
Total & Sistema & 2 & 20.0 & & \\
& 10 & 100.0 & & \\
\hline
\end{tabular}

Fuente: elaboración propia

Como se muestra en la tabla 6 de frecuencias se puede notar que todos los encuestados mencionaron estar más de una hora frente a su computadora, por lo que 4 de ellos dijeron estar 8 horas o más esto representa el $50 \%$ de los encuestados. El resto que también representa el 50\% respondió estar frente a su computadora por menos de 8 horas durante su jornada laboral. Se les recomienda a los empleados tomar medidas precautorias para no presentar algún daño futuro en su sistema visual.

Tabla 7. Descanso: durante su jornada laboral. ¿cuántas horas pasa frente a su computador?

\begin{tabular}{|ll|c|c|c|c|}
\hline & Frecuencia & Porcentaje & $\begin{array}{c}\text { Porcentaje } \\
\text { válido }\end{array}$ & $\begin{array}{c}\text { Porcentaje } \\
\text { acumulado }\end{array}$ \\
\hline Válidos & 2 a 7 horas & 4 & 40.0 & 50.0 & 50.0 \\
& 8 horas o más & 4 & 40.0 & 50.0 & 100.0 \\
& Total & 8 & 80.0 & 100.0 & \\
Perdidos & Sistema & 2 & 20.0 & & \\
Total & & 10 & 100.0 & & \\
\hline
\end{tabular}

Fuente: elaboración propia

En la tabla 7 se puede notar que existe una similitud en las frecuencias de los resultados, ya que la mitad de los encuestados respondieron de manera positiva mientras los restantes lo hicieron de manera contraría, por lo que sólo una persona contestó definitivamente sí sentir molestias visuales mientras que tres más dijeron probablemente si sentir estas molestias, sumadas los porcentajes de estas respuestas nos da como resultado el $50 \%$ de los encuestados. Con base en esto, se infiere que el $50 \%$ restante no siente ninguna molestia visual ya que toma algún tipo de medida ergonómica. 
Tabla 8. Distancia monitor: ¿durante el uso del equipo de cómputo la distancia entre sus ojos y el monitor es de aproximadamente la medida de su antebrazo (entre 50 y $60 \mathrm{~cm}$ )?

\begin{tabular}{|c|c|c|c|c|c|}
\hline & & Frecuencia & Porcentaje & $\begin{array}{c}\text { Porcentaje } \\
\text { válido }\end{array}$ & $\begin{array}{l}\text { Porcentaje } \\
\text { acumulado }\end{array}$ \\
\hline \multirow[t]{3}{*}{ Válidos } & Definitavamente sí & 7 & 70.0 & 87.5 & 87.5 \\
\hline & Probablemente sí & 1 & 10.0 & 12.5 & 100.0 \\
\hline & Total & 8 & 80.0 & 100.0 & \\
\hline \multicolumn{2}{|c|}{ Perdidos Sistema } & 2 & 20.0 & & \\
\hline \multicolumn{2}{|l|}{ Total } & 10 & 100.0 & & \\
\hline
\end{tabular}

Fuente: elaboración propia

En la tabla anterior, se puede apreciar que la mayoría de los encuestados si tienen precaución en cuanto a la distancia que se toman cuando trabajan frente al monitor de su equipo, esta mayoría representa el $87.5 \%$ de los encuestados, se infiere que el resto de las respuestas (12.5\%), se debieron a que los usuarios no conocen las medias adecuadas en cuanto a la distancia adecuada entre los ojos y el monitor.

Tabla 9. Dolores musculares: ¿al usar el equipo de cómputo por un lapso mayor a 2 horas siente dolores musculares?

\begin{tabular}{|ll|c|c|c|c|}
\hline & Frecuencia & Porcentaje & $\begin{array}{c}\text { Porcentaje } \\
\text { válido }\end{array}$ & $\begin{array}{c}\text { Porcentaje } \\
\text { acumulado }\end{array}$ \\
\hline Válidos & Definitavamente sí & 1 & 10.0 & 12.5 & 12.5 \\
& Probablemente sí & 2 & 20.0 & 25.0 & 37.5 \\
& Probablemente no & 4 & 40.0 & 50.0 & 87.5 \\
& Definitavamente no & 1 & 10.0 & 12.5 & 100.0 \\
Perdidos & Total & 8 & 80.0 & 100.0 & \\
Total & Sistema & 2 & 20.0 & & \\
& 10 & 100.0 & & \\
\hline
\end{tabular}

Fuente: elaboración propia

Se puede apreciar en la tabla 9 columna frecuencias que la respuesta de la mayoría de los encuestados se inclinó de manera negativa, este representa el $62.5 \%$ de los cuales $50 \%$ (4 encuestados) dijeron probablemente no sentir ningún dolor muscular, mientras que el $12.5 \%$ dijo definitivamente no sentir ningún dolor muscular, por lo que se infiere que éstos encuestados, si toman medidas ergonómicas en sus puestos de trabajo para evitar estos padecimientos. Por otra parte, la inclinación del 37.5\% de los encuestados fue de manera positiva, ya que dijeron que definitivamente sí o probablemente sí sentir dolores musculares. Este $37.5 \%$ es representado por el $12.5 \%$ 
EST UDIO ERGONÓMICO COMO PARTE DE LA RESPON SABILIDAD SOCIALEN TRABAJADORES DEL CENTRO REGIONAL DE INFORMÁTICA DE LA UNIVERSIDAD VERACRUZANA, MÉXICO

que dijo definitivamente sí sentir dolores musculares y el $25 \%$ que dijeron probablemente sí sentir dolores musculares respectivamente.

Tabla 10. Tipo de dolores musculares: ¿qué tipo de dolores?

\begin{tabular}{|ll|c|c|c|c|}
\hline & Frecuencia & Porcentaje & $\begin{array}{c}\text { Porcentaje } \\
\text { válido }\end{array}$ & $\begin{array}{c}\text { Porcentaje } \\
\text { acumulado }\end{array}$ \\
\hline Válidos & Dolor de cuello & 1 & 10.0 & 33.3 & 33.3 \\
& Dolor de espalda & 1 & 10.0 & 33.3 & 66.7 \\
& Dolor de muñeca & 1 & 10.0 & 33.3 & 100.0 \\
Perdidos & Total & 3 & 30.0 & 100.0 & \\
Total & Sistema & 7 & 70.0 & & \\
& & 10 & 100.0 & & \\
\hline
\end{tabular}

Fuente: elaboración propia

Con base en el cuestionamiento anterior (tabla 9), en donde el $37.5 \%$ correspondiente al $12.5 \%$ ( 1 encuestado) que dijo definitivamente sí sentir dolores musculares y el $25 \%$ ( 2 encuestados) que dijeron probablemente sí sentir dolores musculares.

En la tabla 10 se pide especifiquen el tipo de dolores. Se puede apreciar que los 3 escuentados respondieron estar probablemente o seguros de sentir dolores musculares. Una persona dijo sentir dolor de cuello, que representa el 33.33\% de los encuestados, el siguiente $33.33 \%$ mencionó sentir dolor de espalda y una última, sentir dolor de muñeca que de igual forma representa el $33.33 \%$. Aunque son minoría es inquietante pues ello indica que este personal ignora sobre medidas ergonómicas en el área laboral.

Tabla 11. Mobiliario: ¿se siente cómodo con el mobiliario de su equipo de cómputo (silla y escritorio)?

\begin{tabular}{|ll|c|c|c|c|}
\hline & Frecuencia & Porcentaje & $\begin{array}{c}\text { Porcentaje } \\
\text { válido }\end{array}$ & $\begin{array}{c}\text { Porcentaje } \\
\text { acumulado }\end{array}$ \\
\hline Válidos & Definitivamente sí & 5 & 50.0 & 62.5 & 62.5 \\
& Probablemente sí & 1 & 10.0 & 12.5 & 75.0 \\
& Indeciso & 2 & 20.0 & 25.0 & 100.0 \\
& Total & 8 & 80.0 & 100.0 & \\
Perdidos & Sistema & 2 & 20.0 & & \\
Total & 10 & 100.0 & & \\
\hline
\end{tabular}

Fuente: elaboración propia 
En las frecuencias de la tabla 11, se puede notar que la mayoría de los encuestados dijeron sí sentirse cómodos con el mobiliario de su oficina, lo que representa el $62.5 \%$ de los encuestados, por lo que reduce el riesgo de que los usuarios obtengan problemas de salud derivados de esta, por lo general las respuestas fueron de manera positiva por lo que se infiere que el mobiliario utilizado es el adecuado.

Tabla 12. Mobiliario: ¿su postura al utilizar al equipo de cómputo es, posición de los codos a $90^{\circ}$, rodillas a $90^{\circ}$, cabeza inclinada a $20^{\circ}$ y espalda recta?

\begin{tabular}{|ll|c|c|c|c|}
\hline & Frecuencia & Porcentaje & $\begin{array}{c}\text { Porcentaje } \\
\text { válido }\end{array}$ & $\begin{array}{c}\text { Porcentaje } \\
\text { acumulado }\end{array}$ \\
\hline Válidos & Probablemente sí & 3 & 30.0 & 37.5 & 37.5 \\
& Indeciso & 3 & 30.0 & 37.5 & 75.0 \\
& Probablemente no & 2 & 20.0 & 25.0 & 100.0 \\
& Total & 8 & 80.0 & 100.0 & \\
Perdidos & Sistema & 2 & 20.0 & & \\
Total & 10 & 100.0 & & \\
\hline
\end{tabular}

Fuente: elaboración propia

En la frecuencia de la tabla 12, se observa que es considerable la cantidad de los encuestados que respondieron estar "indecisos" de si su postura es la adecuada al usar el equipo de cómputo (37.5\%), por otra parte, los encuestados que respondieron "Probablemente sî" que representa de igual forma el 37.5\%, lo que manifiesta un desconocimiento por las posturas adecuadas.

Tabla 13. Iluminación: ¿las fuentes de luz como iluminarias y ventanas, provocan problemas de deslumbramientos/reflejos con su monitor?

\begin{tabular}{|ll|c|c|c|c|}
\hline & Frecuencia & Porcentaje & $\begin{array}{c}\text { Porcentaje } \\
\text { válido }\end{array}$ & $\begin{array}{c}\text { Porcentaje } \\
\text { acumulado }\end{array}$ \\
\hline Válidos & Difinitivamente sí & 2 & 20.0 & 25.0 & 25.0 \\
& Probablemente sí & 4 & 40.0 & 50.0 & 75.0 \\
& Probablemente no & 1 & 10.0 & 12.5 & 87.5 \\
& Definitivamente no & 1 & 10.0 & 12.5 & 100.0 \\
& Total & 8 & 80.0 & 100.0 & \\
Perdidos & Sistema & 2 & 20.0 & & \\
Total & 10 & 100.0 & & \\
\hline
\end{tabular}

Fuente: elaboración propia 
En la tabla 13 se aprecia una proporción notable del 50\% en donde los encuestados mencionan que probablemente sí existen problemas de deslumbramiento en sus monitores. El 25\% de los encuestados dijeron si tener problemas de deslumbramientos. Por otra parte, es relativamente una minoría los encuestados que dieron una respuesta con orientación negativa, mencionan que probablemente no o definitivamente no tener problemas de deslumbramiento, esto representa el $25 \%$ conformando este porcentaje en un $12.5 \%$ y $12.5 \%$ respectivamente.

Tabla 14. Medias de ítems

\begin{tabular}{|c|l|c|}
\hline $\mathbf{N}^{\circ}$ & \multicolumn{1}{|c|}{ Ítems } & Media \\
\hline $\mathbf{2}$ & ¿Conoce el significado del término ergonomía? & 4.25 \\
\hline $\mathbf{3}$ & Durante su jornada laboral. ¿Cuántas horas pasa frente a su PC? & 4 \\
\hline $\mathbf{4}$ & $\begin{array}{l}\text { Cuando llega su descanso, o se toma un receso ¿Siente molestia como } \\
\text { irritación, tensión, visión borrosa o enrojecimiento ocular? }\end{array}$ & 3 \\
\hline $\mathbf{5}$ & $\begin{array}{l}\text { ¿Durante el uso del equipo de cómputo la distancia entre sus ojos y el } \\
\text { monitor es de aproximadamente la medida de su antebrazo (entre 50 } \\
\text { y 60 cm)? }\end{array}$ & 4.875 \\
\hline $\mathbf{6}$ & $\begin{array}{l}\text { ¿Al usar el equipo de cómputo por un lapso mayor a 2 horas, siente } \\
\text { dolores musculares? }\end{array}$ & 2.75 \\
\hline $\mathbf{8}$ & $\begin{array}{l}\text { ¿Se siente cómodo con el mobiliario de su equipo de cómputo (silla y } \\
\text { escritorio)? }\end{array}$ & 4.375 \\
\hline $\mathbf{9}$ & $\begin{array}{l}\text { ¿Su postura al utilizar al equipo de cómputo es, posición de los codos } \\
\text { a 90 }{ }^{\circ}, \text { rodillas a 90 }\end{array}$, cabeza inclinada a 20 ${ }^{\circ}$ y espalda recta? & 3.125 \\
\hline $\mathbf{1 0}$ & $\begin{array}{l}\text { ¿Las fuentes de luz como iluminarias y ventanas, provocan problemas } \\
\text { de deslumbramientos/reflejos con su monitor? }\end{array}$ & 3.5 \\
\hline
\end{tabular}

Fuente: elaboración propia

En la tabla 14. Medias de ítems, se observa que lo respondido por los trabajadores es que ellos se encuentran entre indecisos hacia probablemente sí, lo que significa que aplican las medidas ergonómicas.

La distribución de las medias oscila entre los valores 3 "probablemente sî" y 4 "definitivamente sî́" que indican una respuesta positiva favorable.

A partir de la distribución de la media se puede comprobar que la hipótesis es aceptada, tabla 14. Entonces H1 se acepta: En los trabajadores del Centro Regional de Informática se aplican medidas ergonómicas de iluminación y mobiliario en el uso del equipo de cómputo. 
La pregunta de investigación, se responde: Sí, se aplican medidas ergonómicas de iluminación y mobiliario en los trabajadores del CRI cuando hacen uso del equipo de cómputo. Con base en lo anterior, se puede decir que en términos generales el CRI es una empresa Socialmente Responsable, es aceptable en la directriz relación laboral. Como empresa, el CRI procura en el cuidado de la salud de sus trabajadores.

\section{Conclusiones}

La ergonomía es una disciplina que se hace un gran aliado para las organizaciones y las Empresas Socialmente Responsables, gracias a ella se logra mayor productividad en sus trabajadores, en la mejora de la calidad, en eliminar o reducir los riesgos profesionales, reducir los "costos ocultos" resultantes de las deficientes condiciones de trabajo (ausentismo, rotación de la mano de obra, derroche de materias primas y energía, etc.) sin llegar a intensificar el trabajo humano. (Wisner, 1998)

Una inadecuada preparación o deficiencias de las condiciones organizacionales como el lugar de trabajo pueden llevar a una relación hombre-máquina que producen riesgos sutiles en la salud de los trabajadores y que aparecen en el ámbito físico, psicológico y social. Esto se ha estudiado desde comienzos de la década de 1980 acerca de los efectos de incorporar tecnologías computacionales al trabajo, en particular por la Fundación Europea para la Mejora de las Condiciones de Vida y Trabajo ha sido un tema preponderante.

La renovación tecnológica en las oficinas de trabajo se encuentra aún en proceso de desarrollo, y ha sido controvertida debido a sus posibles repercusiones sobre la salud de los trabajadores. De acuerdo con el objetivo primordial de la Responsabilidad Social Empresarial (RSE) en las Organizaciones, se concluye que el Centro Regional de Informática procura e intenta cuidar la relación laboral a través de aplicación de medidas ergonómicas en sus trabajadores, demostrando solidaridad y respeto en el cuidado de la salud. Cada una de las preguntas fueron analizadas y de acuerdo a la variable iluminación, las respuestas de los ítems oscilaron entre los valores 3 a 5, equivalentes a que no tienen problemas con la vista como irritación, vista borrosa, enrojecimiento ocular, aunque pasen gran tiempo frente al monitor y que no sufren por reflejos o deslumbramientos, además mantienen la distancia correcta entre los ojos y la pantalla de la computadora.

En cuanto a la dimensión mobiliario, una mayoría de los trabajadores del CRI no sienten dolores musculares de ningún tipo: cuello, mano, espalda u otro; y que mantienen correctamente su postura al estar sentados trabajando frente a la 
EST UDIO ERGONÓMICO COMO PARTE DE LA RESPONSABILIDAD SOCIAL EN TRABAJADORES DEL CENTRO REGIONAL DE INFORMÁTICA DE LA UNIVERSIDAD VERACRUZANA, MÉXICO

computadora, básicamente en conclusión se sienten cómodos con el mobiliario, el cual es el correcto. De lo contrario, los resultados negativos pueden derivarse enfermedades como: torcimiento del cuello, lumbalgia, lordosis, escoliosis, síndrome del túnel carpiano, miopatías, entre otras.

Por lo anterior, se recomienda a los trabajadores del Centro Regional de Informática tomar posturas correctas frente a su PC en el puesto de trabajo para evitar malestares como dolores musculares. Referente a la iluminación, por lo general en el CRI hay fuentes de luz que provocan problemas de deslumbramientos o reflejos en sus monitores, se recomienda buscar una solución a este asunto para evitar algunos riesgos en la salud, ya que en el caso de la visión se pueden derivar padecimientos serios a tan temprana edad en los trabajadores, como: trastornos visuales que incluyen dolor e inflamación de parpados, pesadez ocular, lagrimeo, enrojecimiento, entre otros; cefalalgias como dolores de cabeza, y la fatiga, la cual da un sensación de falta de energía o agotamiento.

Con base en lo anterior, las empresas y organizaciones socialmente responsables deben incluir los factores de riesgo en la salud derivados de la ergonomía, lo que conlleve de manera sistematizada en cada puesto laboral a revisiones periódicas de los trabajadores que habitualmente usan las computadoras, y además a la adquisición paulatina de mobiliario adecuado como de sus instalaciones.

Los ambientes adecuados y amigables reducen consecuencias negativas en la salud, mediante el cumplimiento de lo regulado en relación con riesgos laborales en salud. Finalmente, tal y como lo ha mencionado Alain Wister en su libro Ergonomía y Condiciones de Trabajo. La ergonomía como parte de la RSE es de gran utilidad sí: "Los empresarios, son los responsables de las áreas funcionales de la empresa y los trabajadores se proponen eliminar o reducir los riesgos profesionales en su misma fuente para evitar accidentes de trabajo y enfermedades profesionales, cuando tratan de mejorar las condiciones de trabajo para evitar el incremento de la fatiga y cuando se desea mejorar la eficiencia de las actividades productivas y de servicios, en cuanto a producción y calidad". 


\section{Referencias Bibliográficas}

ARMS, (2012). Medidas preventivas en el ámbito laboral, postura correcta en la computadora. ARMS México. Recuperado de: http://armsmexico.com/page/6/

Argandoña, Antonio (2012). Otra definición de responsabilidad social. Cátedra "la Caixa" de responsabilidad social de la empresa y el Gobierno corporativo. IESE Business School Universidad de Navarra: Madrid, 1-2.

Asociación Internacional de Ergonomía. (2000). Definición de ergonomía. Recuperado de: http://www.iea.cc/whats/index.html

Cañas, J., y Salmerón L (2004). Sobre la relación entre la ergonomía y psicología cognitivas. Anuario de Psicología, 35(4), 507-519.

Caravedo, Baltazar. (1999). Lo social y la empresa a fines de siglo. Lima, Perú: Centro de Investigación de la Universidad del Pacifico, 1-244.

Carrasquero, E. y Seijo, C. (2009). La ergonomía organizacional y la responsabilidad social inclusiva y preactiva: Un compromiso dentro de los objetivos de la organización. Revista Clío América, 3(6), 183-192.

Correa, M., G. Gallopin y G. Núñez (2005). Medir para gestionar la RSC. Harvard Business Review. Vol. 83 (8), 51-58.

Cuenca, G. (2012). Conceptos básicos de ergonomía. Ergonomía para Empresas. Argentina: Universidad Tecnológica Nacional, Facultad Regional de Buenos Aires, 1-40.

Degelman, C., Hayes, B. y Berkowitz, L. (2005). Active Citizenship today Field Guide. Close Up Foundation. (2a . Ed.).

Delgado, J. (2011). Ergonomía física en obra: lesiones producidas e instrumentos para mejorarla. (Tesis de pregrado). Valencia: Universidad Politécnica de Valencia.

Del Rio, J. y González, M. (2007). Trabajo prolongado con computadoras: Consecuencias sobre la vista y la fatiga cervical. Congreso Internacional de Ergonomía, Ciudad de México. 
EST UDIO ERGONÓMICO COMO PARTE DE LA RESPONSABILIDAD SOCIAL EN TRABAJADORES DEL CENTRO REGIONAL DE INFORMÁTICA DE LA UNIVERSIDAD VERACRUZANA, MÉXICO

Ergocare. (2011).¿Tu postura al trabajar, es la correcta?. Recuperado de: https:// ergocare.wordpress.com/2011/08/26/tu-postura-al-trabajar-es-la-correcta/

Fidalgo, A. (2006). Ordenador-persona: las distancias ideales. Consultoría de usabilidad y diseño centrado en el usuario. Recuperado de: http:// www.usolab.com/wl/2006/04/ordenadorpersona-las-distancia.php

Instituto INSHT. (s.f) Objetivos de la ergonomía. Instituto Nacional de Seguridad e Higiene, España. Recuperado de: http://www.insht.es/InshtWeb/Contenidos/ Documentacion/TextosOnline/GuiasMonitor/Ergonomia/I/Ficheros/ei08.pdf

Jaureguiberry, M. (s.f). Seguridad e higiene en el trabajo. Argentina: Universidad Nacional del Centro de la Provincia, Buenos Aires, 1-9.

Laurig, W. y Vedder, J. (1992). Ergonomía. Madrid: Instituto Nacional de Seguridad e Higiene.

Leirós, L. (2009). Historia de la Ergonomía, o de cómo la Ciencia del Trabajo se basa en verdades tomadas de la Psicología. Revista de historia de la psicología, 30(4), 33-53.

Melo, J. (2003). Ergonomía - Conformación fisiológica del punto de trabajo. Recuperado de: http://www.estrucplan.com.ar/Producciones/imprimir.asp? IdEntrega=391

Muñoz, C y Vanegas, J. (2012). Asociación entre puesto de trabajo computacional y síntomas músculo esqueléticos en usuarios frecuentes. Medicina y seguridad del trabajo, 58 (227), 98-106.

Ramos, A. (2007). Estudio de factores de riesgo ergonómico que afectan el desempeño laboral de usuarios de equipo de cómputo en una institución educativa. (Tesis de Maestría). México: Instituto Politécnico Nacional.

Rodríguez, T. (2013). Estudio en ergonomía informática con enfoque sustentable en la escuela de enfermería campus Coatzacoalcos. (Tesis de pregrado). Coatzacoalcos Veracruz, México: Universidad Veracruzana.

Salud180. (2013). 7 enfermedades oculares por el uso de la tecnología. Recuperado de: http://www.correodelorinoco.gob.ve/impacto/7-enfermedades-ocularespor-uso-tecnologia/ 
Singleton, T. (1982). The body at work: Biological ergonomics. Cambridge: University Press.

Wisner, A. (1998). Ergonomía y Condiciones de Trabajo. Estudios e Investigaciones Laborales de la SECYT, CEIL-CONICET-CN Humanitas. Argentina: Buenos Aires, 1-9. 